\title{
Ethical Obligations of Manuscript Reviewers for Weed Technology
}

Manuscript review is an essential step toward publication. Therefore, every scientist is obligated to do a fair share of reviewing for the scientific method to be effective.

1. Objectively judge the quality of a manuscript, of its experimental and theoretical basis, and of its interpretations and discussion, using accepted scientific and literary standards.

2. The obligation of strict objectivity is especially important either when a manuscript challenges an observation or interpretation that the reviewer has published or when a manuscript is closely related to the reviewer's research. When there is a serious conflict of interest, a reviewer can return the manuscript without review promptly to the editor, advising him of the conflict. Or the reviewer can prepare a signed review stating both his/her direct interest in the research being reported, including any comments or criticism.

3. Explain and support judgments in writing so that editors and authors can understand the basis of reviewer comments. Unsupported claims by reviewers (or by authors in rebuttal) are of little value. Criticisms should be presented constructively to help the author to improve the manuscript. Some manuscripts need extensive revision, including deletion of unacceptable experiments and unsupported conclusions.
4. Alert authors to other scientists' relevant research that was not cited. However, remember that complaints that literature by the reviewer was not cited may seem self serving. Inform the editor of any substantial similarity between the manuscript under review and any published paper or manuscript submitted concurrently to other journal.

5. Act promptly and submit a report within specified time. When circumstances preclude prompt evaluation, return the unreviewed manuscript immediately to the editor. "Excessive delays in the review process area disservice to both authors and readers. Scientific reputations and professional advancement of authors depend, in part, on prompt publication of their research findings. New information should be made available to the scientific community without delay ( $C B E$ Style Manual, 5th edition, p. 4 and 5)."

6. Treat a manuscript as a confidential document. It neither should be shown to nor discussed with others except, in special cases, to consult specialists for specific advice. The identities of those consulted should be disclosed to the editor.

7. Do not use unpublished information or arguments contained in a manuscript under consideration without the author's consent. Until the paper is published, its content and ideas are the author's property. 